\title{
Heterotopic Ossification with and without Neovascularization in Aortic Valves: A Histopathological Study
}

\section{Susumu Matsukuma ${ }^{1,2}$, Ayano Koga ${ }^{1}$, Hiroaki Takeo ${ }^{1}$ and Kimiya Sato ${ }^{1}$}

${ }^{1}$ Department of Pathology Japan Self-Defense Forces Central Hospital, Ikejiri 1-2-24, Setagaya-ku, Tokyo 154-0001, Japan

${ }^{2}$ Health Care Center, Japan Self-Defense Forces Central Hospital, Ilkejiri 1-2-24, Setagaya-ku, Tokyo 154-0001, Japan

\begin{abstract}
Heterotopic ossification can be found in neovascularized aortic valves with end-stage valvular disease, but its histopathological characteristics are not fully understood. The present study found ossification and neovessels in $21(28 \%)$ and $54(72 \%)$ of 75 surgically removed dysfunctional aortic valves, respectively; there was a significant association between them $(P<0.001)$. Fatty bone marrow was found in 5 aortic valves $(6.7 \%)$ and always contained neovessels. Neovessels could be divided into thick-walled, arteriole-like vessels (ThKA-Vs) and thin-walled, capillarylike vessels (ThNC-Vs). ThKA-Vs would be a hallmark of rheumatic disease, were always accompanied by ThNC$V s$, and were closely related to ossification $(P=0.046)$. ThNC-Vs may be in part branches of ThKA-Vs, but were also identified in 23 aortic valves without ThKA-Vs. In 44 non-rheumatic dysfunctional aortic valves, such ThNC-Vs only were also associated with ossification $(P=0.004)$. In addition, the present study revealed vessel-independent focal/ minute ossification without nearby neovessels in 6 dysfunctional aortic valves, and also in 4 of 75 age- and sexmatched postmortem non-dysfunctional aortic valves with no neovessels or prominent calcification/thickening; there were no significant differences in the incidence between them. Neovascularized vessels would consist of rheumatic neovessels (ThKA-Vs and their branches) and non-rheumatic neovessels (ThNC-Vs only), and the presence of neovessels is one of the risk factors for aortic valvular ossification. However, aortic valvular ossification can occur in various pathogeneses, such as rheumatic neovessel-dependent ossification, non-rheumatic neovessel-dependent ossification, and rarely vessel-independent focal/minute ossification.
\end{abstract}

Keywords: Aortic valve; Heterotopic ossification; Bone; Neovascularization; Calcification; Rheumatic disease

Abbreviations: EVG: Elastica Van Gieson; H\&E: Hematoxylin and Eosin; ThKA-Vs: Thick-walled Arteriole-like Vessels; ThNC-Vs: Thinwalled Capillary-like Vessels

\section{Introduction}

Heterotopic ossification in aortic valves usually involves calcified valves with end-stage valvular disease [1-5]. Ossification in calcified aortic valves has been considered to be a partly metaplastic process similar to enchondral or endochondral ossification [1-4] accompanied by vessel proliferation $[1,6,7]$. Aortic valve itself is avascular $[3,5,8]$ but neovascularization develops in postinflammatory or rheumatic aortic valves [8-11]. Indeed, the relationship between aortic valvular ossification and neovessels has been suggested [1-3]. However, the detailed pathological features of aortic valvular ossification have not been fully elucidated because 1) previous examinations of aortic valvular ossification in larger series were mixed together with those in mitral valves $[1,2]$; 2) the neovessels have been mentioned in non-rheumatic aortic valves [5,12]; and 3) the illustrated neovessels appeared to be divided into thick-walled and thin-walled vessels $[5,9]$. In addition, we speculated that ossification may occur in non-neovascularized aortic valves because ossification can be found in an avascular condition, such as intra-articular free bodies without blood supply $[13,14]$. In this study, focusing on the presence and the histology of neovascularization, we examined ossification in surgically excised dysfunctional aortic valves. For a control study, postmortem non-dysfunctional aortic valves were also histologically examined.

\section{Materials and Methods}

A total of 75 surgically removed dysfunctional aortic valves, excluding infectious endocarditic valves, were retrieved from the surgical pathology files of the Department of Pathology, Japan Self-
Defense Forces Central Hospital (1987-2013), including 65 tricuspid and 10 bicuspid aortic valves, and were examined. The patients were 46 men and 29 women with a mean age of 71.3 years (range, 50-88 years), and consisted of 35 with aortic stenosis, 24 with aortic regurgitation, and 16 with combined AS and AR. In 60 tricuspid and 10 bicuspid valves, classified cusps were separately cut into 2-12 sections with free edges and cuspal base. In the other 5 tricuspid valves, unclassified cusps were cut into 1-18 sections. The total number of sections in each valve ranged from 6 to 26 (mean, 16.9). All were fixed with 10-20\% buffered formalin and calcified valves were decalcified using formic acid. Four- $\mu \mathrm{m}$-thick sections were stained with hematoxylin and eosin (H\&E) and elastica van Gieson (EVG). The neovessels were divided into thick-walled, arteriole-like vessels (ThKA-Vs) and thin-walled, capillary-like vessels (ThNC-Vs). Clinical findings were available from the medical charts and/or the request forms of surgical pathology examinations. According to the proposed criteria [8-10], 75 dysfunctional aortic valves were classified into 31 rheumatic aortic valves and 44 non-rheumatic aortic valves ( 24 nodular calcific tricuspid aortic valves, 3 congenital bicuspid aortic valves, 15 regurgitant floppy aortic valves, and 2 regurgitant aortic valves with other causes). The associations of ossification with

*Corresponding author: Susumu Matsukuma, Health Care Center, Japan Self-Defense Forces Central Hospital, 1-2-24 Ikejiri, Setagaya-ku, Tokyo 1540001, Japan, Tel: +81-3-3411-0151 Ext. 6446; Fax: +81-3-3418-0197; E-mail: skuma@cocoa.plala.or.jp

Received August 21, 2014; Accepted September 16, 2014; Published September 16, 2014

Citation: Matsukuma S, Koga A, Takeo H, Sato K (2014) Heterotopic Ossification with and without Neovascularization in Aortic Valves: A Histopathological Study. J Cytol Histol 5: 279. doi:10.4172/2157-7099.1000279

Copyright: (c) 2014 Matsukuma S, et al. This is an open-access article distributed under the terms of the Creative Commons Attribution License, which permits unrestricted use, distribution, and reproduction in any medium, provided the original author and source are credited. 
Citation: Matsukuma S, Koga A, Takeo H, Sato K (2014) Heterotopic Ossification with and without Neovascularization in Aortic Valves: A Histopathological Study. J Cytol Histol 5: 279. doi:10.4172/2157-7099.1000279

Page 2 of 5

clinicopathological findings in each case were statistically analyzed using chi-square test, Fisher's exact test, Mann-Whitney U-test, and unpaired t-test. Statistical significance was set at $P<0.05$. In addition, to assess the relationship between each cuspal ossification and neovessels, the histological features were evaluated in each cusp with ossification. For a control study, 75 age- and sex-matched postmortem tricuspid aortic valves from patients without aortic valvular dysfunction and infectious endocarditis, composed of 46 men and 29 women with a mean age of 71.3 (range, 50-88), were retrieved from the autopsy files of the Department of Pathology, Japan Self-Defense Forces Central (2008-2012), and were examined. Postmortem interval ranged from 1 to 36 hours (mean, 9.9 hours). Each cusp was cut into 1-7 sections and stained with H\&E and EVG. The number of valvular sections in each case ranged from 3 to 20 (mean, 15.3).

\section{Results}

Neovessels and heterotopic ossification were found in $54(72.0 \%)$ and $21(28.0 \%)$ of 75 dysfunctional aortic valves, respectively. Ossification was more common in rheumatic aortic valves (13 of $31,41.9 \%)$ than in non-rheumatic ones ( 8 of $44,18.2 \%)(P=0.046)$. Ossification usually consisted of rim-like small lamellar bones in the peripheral regions of calcified lipid or atheromatous materials, frequently accompanied by nearby neovessels (Figure 1a). Ossification was highlighted red by EVG, which was discriminated from calcified lipid (Figure 1a, inset). Scattered ThKA-Vs were found in 31 dysfunctional valves (41.3\%), continuously connected to the cuspal base, and were always concomitant with ThNC-Vs. ThNC-Vs were occasionally aggregated in an insular fashion (Figure 1b), and were also identified in 23 dysfunctional valves without ThKA-Vs. These 23 dysfunctional valves with ThNC-Vs only included 21 nodular calcific tricuspid aortic valves ( $87.5 \%$ of the cases),
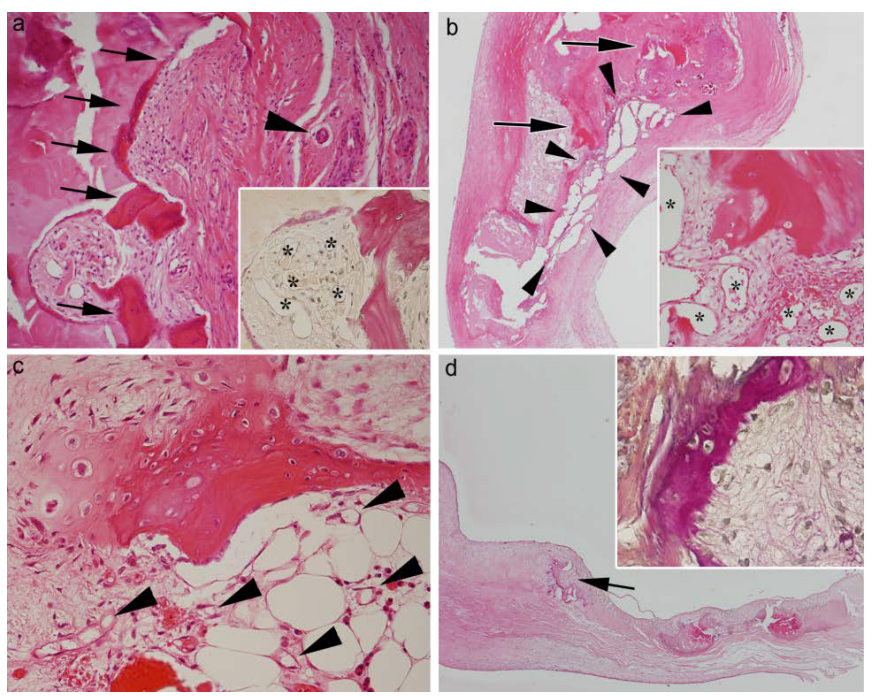

Figure 1: Heterotopic ossification in dysfunctional aortic valves. (a) Rimlike ossification (arrows) in calcified atheroma, accompanied by thick-walled arteriole-like neovessel (arrowhead), and a high-power view (inset) of ossification stained red by EVG and nearby thin-walled capillary-like vessels (asterisks). (b) Heterotopic ossification (arrows) and aggregated thin-walled vessels (arrowheads) in a nodular calcific tricuspid aortic valve without thickwalled vessels, and a high-power view (inset) showing bone and nearby thinwalled vessels (asterisks). (c) Enchondral ossification composed of bone and fatty marrow adjacent to metaplastic cartilage accompanied by thin-walled vessel proliferation (arrowheads). (d) Focal and minute ossification (arrow) in calcified lesions without nearby neovessels and a high-power view (inset) of thin rim-like bone stained red by EVG adjacent to edematous stroma without vessels.

\begin{tabular}{|l|c|c|c|}
\hline & $\begin{array}{c}\text { AVs with HO } \\
(\mathbf{n = 2 1 )}\end{array}$ & $\begin{array}{c}\text { AVs without HO } \\
(\mathbf{n = 5 4 )}\end{array}$ & $\boldsymbol{P}$-value \\
\hline Age (y), range (mean) & $52-84(70.8)$ & $50-88(71.5)$ & $0.845^{\star}$ \\
\hline Gender (male/female) & $15 / 6$ & $31 / 23$ & $0.392^{\dagger}$ \\
\hline Moderate to severe calcification & 21 & 27 & $<0.001^{\ddagger}$ \\
\hline Moderate to severe fibrous thickening & 18 & 17 & $<0.001^{\ddagger}$ \\
\hline Neovessels (ThKA-Vs and/or ThNC-Vs) & 21 & 33 & $<0.001^{\ddagger}$ \\
\hline \multicolumn{1}{|c|}{ ThKA-Vs } & 13 & 18 & $0.046^{\dagger}$ \\
\hline \multicolumn{1}{|c|}{ ThNC-Vs only } & 8 & 15 & $0.554^{\ddagger}$ \\
\hline Foamy cell aggregation & 10 & 17 & $0.299^{\dagger}$ \\
\hline Lymphoplasmacytic infiltration & 19 & 18 & $<0.001^{\ddagger}$ \\
\hline $\begin{array}{l}\text { Metaplastic cartilage (hyaline and/or } \\
\text { fibrous cartilage) }\end{array}$ & 10 & 3 & $<0.001^{\ddagger}$ \\
\hline
\end{tabular}

AVs: aortic valves; HO: heterotopic ossification; ThNC-Vs: thin capillary vessel-like neovessels; ThKA-Vs: thick arteriole-like neovessels.

"Mann-Whitney U test.

${ }^{\dagger}$ Chi-square test.

‡Fisher's exact test.

Table 1: Clinicopathological comparison of 75 dysfunctional aortic valves with and without heterotopic ossification

\begin{tabular}{|l|c|c|c|}
\hline & $\begin{array}{c}\text { Non-rheumatic AVs } \\
\text { with HO (n=8) }\end{array}$ & $\begin{array}{c}\text { Non-rheumatic AVs } \\
\text { without HO (n=36) }\end{array}$ & P-value \\
\hline Age (y), range (mean) & $67-84(76.1)$ & $52-88(73.9)$ & $0.558^{*}$ \\
\hline Gender (male/female) & $4 / 4$ & $20 / 16$ & $0.915^{\dagger}$ \\
\hline $\begin{array}{l}\text { Moderate to severe } \\
\text { calcification }\end{array}$ & 8 & 17 & $0.007^{\ddagger}$ \\
\hline $\begin{array}{l}\text { Moderate to severe fibrous } \\
\text { thickening }\end{array}$ & 7 & 9 & $0.002^{\ddagger}$ \\
\hline ThNC-Vs only & 8 & 15 & $0.004^{\ddagger}$ \\
\hline Foamy cell aggregation & 3 & 8 & $0.391^{\dagger}$ \\
\hline $\begin{array}{l}\text { Lymphoplasmacytic } \\
\text { infiltration }\end{array}$ & 6 & 9 & $0.013^{\ddagger}$ \\
\hline $\begin{array}{l}\text { Metaplastic cartilage } \\
\text { (fibrous and/or hyaline } \\
\text { cartilage) }\end{array}$ & 3 & 2 & $0.039^{\ddagger}$ \\
\hline
\end{tabular}

AVs: aortic valves; HO: heterotopic ossification; ThNC-Vs: thin capillary vessel-like neovessels; ThKA-Vs: thick arteriole-like neovessels.

"Unpaired t-test.

${ }^{\dagger}$ Chi-square test.

¥Fisher's exact test.

Table 2: Clinicopathological comparison of 44 non-rheumatic dysfunctional aortic valves with and without heterotopic ossification.

1 congenital bicuspid aortic valve, and 1 regurgitant aortic valve due to aortitis. Fatty bone marrow without hematopoietic cells (Figure 1c) was found in 5 valves (6.7\%), and was invariably accompanied by neovessels. The association of ossification with other features in 75 dysfunctional aortic valves is summarized in Table 1. Ossification was associated with moderate to severe calcification, moderate to severe fibrous thickening, neovessels (ThKA-Vs and/or ThNC-Vs), lymphoplasmacytic infiltration, metaplastic cartilage (hyaline and/or fibrous cartilage) (all, $P<0.001)$, and ThKA-Vs $(P=0.046)$, but not with age, gender, ThNC-Vs only, and foamy cell aggregation. Regarding 44 non-rheumatic dysfunctional aortic valves (Table 2), ossification was closely related to ThNC-Vs only $(P=0.004)$, together with moderate to severe calcification $(P=0.007)$, moderate to severe fibrous thickening $(P=0.002)$, lymphoplasmacytic infiltration $(P=0.013)$, and cartilage $(P=0.039)$. Ossification was present in $18(56.3 \%)$ of 32 prominently calcified and thickened dysfunctional aortic valves (coexisting with both moderate to severe calcification and moderate to severe fibrous thickening).

Twenty-one ossified dysfunctional aortic valves consisted of 1 unclassified tricuspid valve and 20 classified valves (6 bicuspid and 
Citation: Matsukuma S, Koga A, Takeo H, Sato K (2014) Heterotopic Ossification with and without Neovascularization in Aortic Valves: A Histopathological Study. J Cytol Histol 5: 279. doi:10.4172/2157-7099.1000279

Page 3 of 5

14 tricuspid valves). Ossification was found without exception in 33 classified cusps and involved $\geq 2$ cusps within each aortic valve in 11 (55.0\%) of the 20 classified valves (Table 3 ). Regarding the 14 classified tricuspid aortic valves, ossification was distributed in 6 left coronary cusps, 7 right coronary cusps, and 11 non-coronary cusps; there were no favored cusps of ossification among these 3 cusps $(P=0.279)$. Table 4 summarizes the histopathological features of the 33 classified cusps with ossification. Ossification was focal and multifocal in $9(27.3 \%)$ and 24 cusps (72.7\%), respectively. Thirty-two ossified cusps (97\%) were neovascularized. In 27 of them, ossification was always found concomitant with nearby neovessels, and mostly (in 24 cusps) multifocal. However, in the other 5, ossification was focal and minimal, and was not accompanied by nearby vessels, suggesting vessel-independent ossification (Figure 1d), although ThNC-Vs were identified in areas distant from ossified lesions. Foamy cells aggregated adjacent to the vessel-independent ossification in cusp only. Focal and minute vesselindependent ossification was also observed in 1 non-neovascularized cusp, but was not accompanied by foamy cells. These 6 cusps exhibiting vessel-independent ossification belonged to 6 classified aortic valves ( 6 of 70 classified dysfunctional valves, $8.6 \%$ ), all of which were nodular calcific tricuspid aortic valves with aortic stenosis/combined aortic stenosis and regurgitation. Lymphoplasmacytic infiltration near ossified lesions was observed in 24 cusps $(72.7 \%)$, and invariably coexisted with ThNC-Vs. Seventeen ossified cusps had metaplastic cartilage, and 10 of them showed the transition between cartilage and bone. In 9 of 10 cusps showing ossification from cartilage, there were enchondral

\begin{tabular}{|l|c|c|}
\hline & $\begin{array}{c}\text { The number of cusps } \\
\text { involved by HO }\end{array}$ & $\begin{array}{c}\text { The number } \\
\text { of AVs }\end{array}$ \\
\hline Total classified AVs with $\mathrm{HO}(\mathrm{n}=20)$ & 1 cusp only & 9 \\
\hline & $\geq 2$ cusps & 11 \\
\hline Classified tricuspid AVs with $\mathrm{HO}(\mathrm{n}=14)$ & 1 cusp only & 6 \\
\hline & 2 cusps & 6 \\
\hline & All 3 cusps & 2 \\
\hline Classified bicuspid AVs with $\mathrm{HO}(\mathrm{n}=6)$ & 1 cusp only & 3 \\
\hline & All 2 cusps & 3 \\
\hline
\end{tabular}

AV: aortic valve; HO: heterotopic ossification

Table 3: The number of cusps involved by heterotopic ossification in each case on 20 classified aortic valves.

\begin{tabular}{|c|l|}
\hline & $\begin{array}{l}\text { n (\% of 33 classified } \\
\text { cusps with HO) }\end{array}$ \\
\hline Histopathological features of HO & \\
\hline Focal and minute HO/multifocal HO & $9(27.3 \%) / 26(72.7 \%)$ \\
\hline Enchondral ossification-like features from cartilage & $9(27.3 \%)$ \\
\hline Histopathological features in the background of cusps with HO & \\
\hline Neovessels (ThKA-Vs and/or ThNC-Vs) & $32(97.0 \%)$ \\
\hline Metaplastic cartilage (hyaline and/or fibrous cartilage) & $17(51.5 \%)$ \\
\hline Histopathological features near/adjacent to HO & \\
\hline Neovessels (ThKA-Vs and/or ThNC-Vs) & $27(81.8 \%)$ \\
\hline No distinct neovascularized vessels & $6(18.2 \%)^{*}$ \\
\hline Lymphoplasmacytic infiltration & $24(72.7 \%)^{\dagger}$ \\
\hline Metaplastic cartilage (hyaline and/or fibrous cartilage) & $10(30.3 \%)^{\ddagger}$ \\
\hline
\end{tabular}

HO: heterotopic ossification; ThKA-Vs: thick, arteriole-like neovascularized vessels; ThNC-Vs: thin capillary-like neovascularized vessels.

${ }^{*}$ Including 1 non-neovascularized cusp and 5 cusps in which neovascularization was present in areas distant from $\mathrm{HO}$.

${ }^{\dagger}$ All were found in neovascularized stroma.

FIncluding 9 cusps with enchondral ossification-like features and 1 cusp with "vessel-independent heterotopic ossification" from cartilage.

Table 4: Histopathological features of 33 aortic valvular cusps with heterotopic ossification.

\begin{tabular}{|l|c|c|c|}
\hline & $\begin{array}{c}\text { Postmortem AVs } \\
\text { with HO (n=4) }\end{array}$ & $\begin{array}{c}\text { Postmortem AVs } \\
\text { without HO (n=71) }\end{array}$ & $\boldsymbol{P}$-value \\
\hline Age (y), range (mean) & $64-86(76.3)$ & $50-88(71.0)$ & $0.308^{*}$ \\
\hline Gender (male/female) & $3 / 1$ & $43 / 28$ & $0.961^{\dagger}$ \\
\hline Moderate calcification & 1 & 5 & $0.289^{\ddagger}$ \\
\hline Foamy cell aggregation & 4 & 38 & $0.034^{\ddagger}$ \\
\hline Lymphoplasmacytic infiltration & 0 & 4 & $0.512^{\dagger}$ \\
\hline
\end{tabular}

AVs: aortic valves; HO: heterotopic ossification; ThNC-Vs: thin capillary vessel-like neovessels; ThKA-Vs: thick arteriole-like neovessels.

Unpaired t-test.

+Chi-square test.

‡Fisher's exact test.

Table 5: Clinicopathological comparison of 75 non-dysfunctional postmortem aortic valves with and without heterotopic ossification.

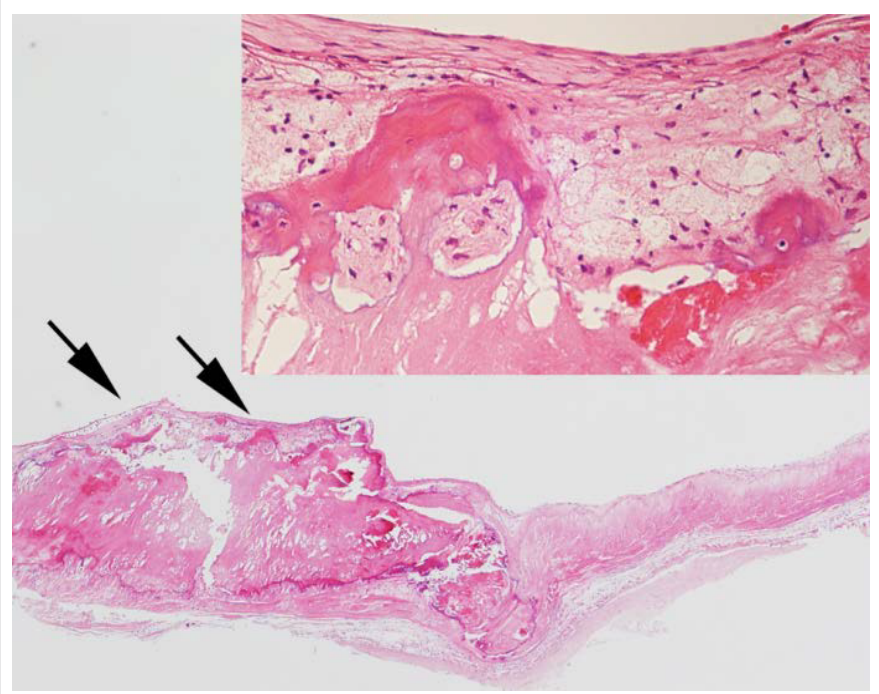

Figure 2: Vessel-independent heterotopic ossification (arrow) in mildly to moderately calcified non-dysfunctional postmortem aortic valve. A high power view (inset) showing rim-like bones accompanied by aggregated foamy cells but without neovascularization.

ossification-like features accompanied by nearby ThNC-Vs, but in the other 1, vessel-independent ossification from cartilage was suggested because of a lack of nearby vessels.

In 75 age- and sex-matched control non-dysfunctional aortic valves, neovessels, severe calcification, moderate to severe fibrous thickening, and cartilage were not found, although moderate calcification, lymphoplasmacytic infiltration, and foamy cell aggregation were observed in $6(8.0 \%), 4(5.3 \%)$, and 42 valves (56.0\%), respectively. In 4 non-dysfunctional valves (5.3\%), focal and minute vessel-independent ossification was found, and involved 1 cusp only in each valve (left coronary cusp in 1 , right coronary cusp in 1 , and non-coronary cusps in 2 non-dysfunctional aortic valves). Ossification in non-dysfunctional aortic valves was significantly associated with foamy cell aggregation $(P=0.034)$, but not with age, gender, moderate calcification, or lymphoplasmacytic infiltration (Table 5). Aggregated foamy cells were found adjacent to ossified lesions (Figure 2) in 2 non-dysfunctional valves (50\%), but nearby lymphoplasmacytic infiltration was not found.

\section{Discussion}

This study found neovessels in $72 \%$ of dysfunctional aortic valves, and divided them into ThKA-Vs and ThNC-Vs, according to the previously reported features $[5,9]$. ThKA-Vs were continuously found 
with the cuspal base and would be identical to a histological marker of rheumatic aortic valves [9]. ThKA-Vs were invariably concomitant with ThNC-Vs, and ThNC-Vs may be in part the branches of ThKA-Vs. However, ThNC-Vs were also identified in 23 dysfunctional aortic valves without ThKA-Vs. These findings suggest that the neovessels would be composed of "rheumatic neovessels" (ThKA-Vs and their branches) and "non-rheumatic neovessels" (ThNC-Vs only). Non-rheumatic neovessels could be found in stenotic aortic valves $[5,12,15]$ and would be induced by rheumatic disease-unrelated inflammation, such as endothelial injury, microthrombosis, plaque rapture or hemorrhage $[3,12,15]$. In addition, in our study, non-rheumatic neovessels were identified most frequently in nodular calcific tricuspid aortic valves, accounting for $21(88 \%)$ of the 24 cases. Non-rheumatic neovessels were occasionally aggregated, simulating the localized organization of microhematoma, microthrombi, or degenerated atheromatous lesions.

In the present study, heterotopic ossification was identified in $28 \%$ of dysfunctional aortic valves. Ossification was limited to 1 cusp in $45 \%$ of classified dysfunctional valves with ossification, and was focal and minute in $27 \%$ of 33 classified cusps with ossification. Therefore, the detection of ossification would be influenced by the number of aortic valvular sections examined, possibly contributing to a higher incidence than previously reported $(8-13 \%)[1,2,4,5]$, although the number of valvular sections examined was not described in previous studies. In addition, in our study, ossification was common (56\%) in the prominently calcified/thickened dysfunctional aortic valves, and was more often in dysfunctional valves than in non-dysfunctional valves ( $28 \%$ versus $5 \%)$, similar to the previously emphasized common presence of ossification in end-stage aortic valvular disease [1,4].

The present study demonstrated the close association of ossification with neovessels in aortic valves, consistent with previous findings [1-3]. Fatty bone marrow in 5 dysfunctional aortic valves always contained neovessels, suggesting that bone marrow formation requires neovascularization. Rheumatic neovessels in aortic valves were associated with ossification, related to the higher incidence of ossification in rheumatic aortic valves than that in non-rheumatic aortic valves ( $42 \%$ versus $18 \%$ ). Non-rheumatic neovessels (ThNCVs only) were also associated with ossification in 44 non-rheumatic dysfunctional aortic valves. Lymphoplasmacytic infiltration, which was associated with aortic valvular ossification, was always admixed with ThNC-Vs in each cusp. A similar correlation between thinwalled neovessels and abundant lymphocytes has also been described previously [5]. These findings denoted that progressive aortic valvular dysfunction, significant calcification, marked fibrous thickening, neovessels, and lymphoplasmacytic infiltration are risk factors for aortic valvular ossification, which would interact with each other. Similar relationship between ossification and inflammation has been reported in calcified arterial atherosclerotic plaques $[16,17]$.

The current study also revealed vessel-independent focal/minute ossification in $9 \%$ of classified dysfunctional aortic valves and in $5 \%$ of non-dysfunctional aortic valves without considerable histological changes; there were no significant differences between them $(P=0.743)$. These findings suggest that vessel-independent ossification rarely occurs in aortic valves not associated with the currently proposed risk factors. This process may be associated with foamy cell aggregation in non-neovascularized non-dysfunctional aortic valves, although the statistical analysis on dysfunctional aortic valves could not demonstrate their relationship. On the other hand, vessel-dependent ossification was usually multifocal and involved $\geq 2$ cusps in each case. Therefore, vessel-dependent ossification is not a locally limited event and might be influenced by "osteoblastic phenotype"-related bioactive factors, such as bone morphogenic protein, and Cbfa1 $[1,3,11]$.

Metaplastic cartilage was significantly related to ossification in dysfunctional aortic valves, and was identified in $>50 \%$ of ossified cusps. Hence, the presence of metaplastic cartilage in aortic valves would also be one of the risk factors for ossification. However, in the present study of 33 ossified cusps, enchondral ossification-like features were limited to $8(24 \%)$ and vessel-independent ossification from cartilage to $1(3 \%)$

\section{Limitation}

This study included a relatively low number of cases. Pharmacological anamneses were unknown in this study. In some surgical cases, aortic valvular specimens were not classified in each cusp, and the number of their sections was relatively low. Hence, sampling error in the histological detection of minute ossification or other lesions might be possible. We believe that the current study provided insightful results regarding aortic valvular ossification. However, to confirm our suggestion, further studies of larger series including multivariate analysis and molecular investigation are needed.

\section{Conclusion}

Neovessels are one of the risk factors for aortic valvular ossification. However, heterotopic ossification in aortic valves would be caused by various pathogeneses, including rheumatic vessel-dependent ossification, non-rheumatic vessel-dependent ossification, and rarely vessel-independent ossification.

\section{Acknowledgements}

The authors thank Tomoyuki Hoshi, Takeyuki Obara, and Kenji Okada for excellent technical assistance, and Daniel Mrozek for editing the manuscript.

\section{References}

1. Mohler ER 3rd, Gannon F, Reynolds C, Zimmerman R, Keane MG, et al. (2001) Bone formation and inflammation in cardiac valves. Circulation 103: 1522-1528.

2. Steiner I, Kasparová $P$, Kohout $A$, Dominik J (2007) Bone formation in cardiac valves: a histopathological study of 128 cases. Virchows Arch 450: 653-657.

3. Li C, Xu S, Gotlieb Al (2013) The progression of calcific aortic valve disease through injury, cell dysfunction, and disruptive biologic and physical force feedback loops. Cardiovasc Pathol 22: 1-8.

4. Egan KP, Kim JH, Mohler ER 3rd, Pignolo RJ (2011) Role for circulating osteogenic precursor cells in aortic valvular disease. Arterioscler Thromb Vasc Biol 31: 2965-2971.

5. Mazzone A, Epistolato MC, Gianetti J, Castagnini M, Sassi C, et al. (2006) Biologic features (inflammation and neoangiogenesis) and atherosclerotic risk factors in carotid plaques and calcified aortic valve stenosis. Two different sites of the same disease? Am J Clin Pathol 126: 494-502.

6. Rosenberg AE, Roth SI (2012) Bone: Histology for pathologists, Mills SE ed (4th edn), Lippincott Williams \& Wilkins, Philadelphia.

7. McCarthy EF, Frassica FJ (1998) Pathology of bone and joint disorders with clinical and radiographic correlation. WB Saunders, Philadelphia.

8. Schoen FJ, Sutton MS (1987) Contemporary issues in the pathology of valvular heart disease. Hum Pathol 18: 568-576.

9. Virmani R, Burke A, Farb A, Atkinson JB (2001) Cardiovascular pathology: Major problems in pathology, LiVolsi VA ed (2nd edn), W.B. Saunders, Philadelphia.

10. Chuangsuwanich $T$, Warnnissorn $M$, Leksrisakul $P$, Laksanabunsong $P$, Thongcharoen P, et al. (2004) Pathology and etiology of 110 consecutively removed aortic valves. J Med Assoc Thai 87: 921-934.

11. Rajamannan NM, Nealis TB, Subramaniam M, Pandya S, Stock SR, et al (2005) Calcified rheumatic valve neoangiogenesis is associated with vascula endothelial growth factor expression and osteoblast-like bone formation. Circulation 111:3296-3301. 
Citation: Matsukuma S, Koga A, Takeo H, Sato K (2014) Heterotopic Ossification with and without Neovascularization in Aortic Valves: A Histopathological Study. J Cytol Histol 5: 279. doi:10.4172/2157-7099.1000279

Page 5 of 5

12. Soini Y, Salo T, Satta $J$ (2003) Angiogenesis is involved in the pathogenesis of nonrheumatic aortic valve stenosis. Hum Pathol 34: 756-763.

13. Milgram JW (1977) The development of loose bodies in human joints. Clin Orthop Relat Res : 292-303.

14. Matsukuma S, Takeo H, Okada K, Sato K (2012) Fatty lesions in intra-articular loose bodies: a histopathological study of non-primary synovial chondromatosis cases. Virchows Arch 460: 103-108.

15. Akahori H, Tsujino T, Naito Y, Matsumoto M, Lee-Kawabata M, et al. (2011)
Intraleaflet haemorrhage is associated with rapid progression of degenerative aortic valve stenosis. Eur Heart J 32: 888-896.

16. Niccoli Asabella A, Ciccone MM, Cortese F, Scicchitano P, Gesualdo M, et al. (2014) Higher reliability of 18 F-FDG target background ratio compared to standardized uptake value in vulnerable carotid plaque detection: a pilot study. Ann Nucl Med 28: 571-579.

17. Scicchitano P, Marzullo A, Ciccone MM (2014) The role of intimal arterial calcification in the context of atherosclerotic plaque stability. J Cytol Histol 5: e111. 